\title{
Arnold, Klaus/Preston, Paschal/Kinnebrock, Susanne (Hrsg.): The Handbook of European Communication History
}

\author{
Hoboken, NJ: Wiley Blackwell 2020. 494 Seiten. Preis: 103,01 $€$
}

\section{Maria Löblich}

Angenommen: 9. Dezember 2020 / Online publiziert: 21. Dezember 2020

(C) Der/die Autor(en) 2020

In dieser Zeitschrift ist vor ein paar Monaten eine ,tiefe Internationalisierung“ des Fachs gefordert worden. Die Kritik lautete: Forschungsergebnisse über Kommunikation auf der Welt stammten fast ausschließlich aus westlicher Feder und würden mit westlichem Wissen produziert. Das führe zu Undifferenziertheit und Schieflagen, zu vorschnellen „Generalerzählungen mit Universalitätsanspruch.“ Das Positionspapier forderte „Ent-Westernisierung“ und Dezentrierung in der internationalen Kommunikationsforschung statt ,globaler Injektionen“. Das vorgeschlagene Rezept: Interdisziplinarität und interregionale Teamarbeit.

Es ist aussichtsreich, diese Diskussion auch in der Kommunikationsgeschichte zu führen. Das vorliegende Handbuch bietet dazu eine Gelegenheit, denn das Herausgeberteam aus zwei Deutschen und einem Iren hatte sich ganz im Sinn der tiefen Internationalisierung vorgenommen, dominante westliche, vor allem britische und US-amerikanische Perspektiven zu hinterfragen. Die ,much greater diversity of (hi-)stories from other geo-cultural and socio-economic settings as well as the differing political regions of Europe and the wider world" seien zu berücksichtigen. Auch die Kommunikationsgeschichtsschreibung und ihre unterschiedlich verteilten Ressourcen und Sichtbarkeiten im heutigen Europa sind Grund, um diese Gelegenheit zu nutzen. Was also bietet der Band und was sind seine Erkenntnispositionen in Sachen Raum und Kultur?

Das Buch ist mit der Gründungsgeschichte der ECREA Communication History Section verbunden. Die Idee damals, 2009, lautete: Die Mitglieder der Section tragen eine Kommunikationsgeschichte zusammen, ,,a common European communication history despite Europe’s diversity“. Das Herkunftsspektrum der fast 80 Menschen,

Dr. M. Löblich $(\bowtie)$

Institut für Publizistik- und Kommunikationswissenschaft, Freie Universität Berlin,

Garystraße 55, 14195 Berlin, Deutschland

E-Mail: maria.loeblich@fu-berlin.de 
die an diesem Handbuch mitgearbeitet haben, reicht von Russland bis Irland, von Skandinavien über Tschechien bis Portugal. Mehr noch: der Wissensaustausch zwischen unterschiedlichen europäischen Räumen ist dadurch gesichert worden, dass fast alle 25 Kapitel von interkulturell zusammengesetzten Teams geschrieben wurden. So ist ein Band entstanden, der von seinen Autoren und Autorinnen her ,tief europäisiert“ ist. Der Band lässt sich deshalb als innovatives Wissensprojekt lesen, sowohl personell als auch thematisch, wie gleich noch gezeigt wird. Zugleich bildete er ein Mittel der Vernetzung, Identitätsbildung und Bindung innerhalb der Fachgesellschaft.

Die Kapitel lassen sich mit der Frage zusammenfassen, wie Medieninstitutionen, Journalismus, Nutzung und Öffentlichkeiten in Europa von 1900 bis zur Gegenwart gesellschaftliche Prozesse angetrieben haben und wie sie zugleich andersherum von Trends wie Demokratisierung, Polarisierung oder Wirtschaftskonzentration gestaltet worden sind. Die Beiträge behandeln immer einen Aspekt (häufig eine Mediengattung oder ein Mediensystem) und beschreiben seine Entwicklung für etwa vier bis fünf, variierend ausgesuchte Länder.

Der Band enthält vier Teile. Die ersten drei zergliedern das lange 20. Jahrhundert. Im ersten Teil wird das Aufkommen der Massenkommunikation bis zum Ende des Zweiten Weltkriegs beschrieben. Man kann hier etwa nachlesen, wie sich der europäische Film im Schatten Hollywoods entwickelt hat oder welche Folgen die Russische Revolution für die Etablierung des gelenkten Mediensystems in Russland hatte. Der zweite Teil zum „,binären Europa“ bietet - nur um wenige Beispiele zu nennen Beiträge zur Medienkontrolle in Ost- und Südeuropa oder zur Mediengeschichte des Ost-West-Konflikts. Im dritten Teil kann man die letzten 30 Medienjahre seit Ende des Kalten Kriegs nachlesen, so den Aufstieg multinationaler Konzerne oder die „multiplen Post-Kommunismen“, die in den Mediensystemen Zentral- und Osteuropas zu beobachten waren. Der vierte Teil bündelt zeitlich quer liegende ,Themen und Trends“, etwa Kommunikationsgeschichten von Minderheiten, Gender oder Solidarität. Die Beiträge sind in sich nach Ländern untergliedert oder haben nach einer quer liegenden Gliederungslogik gesucht. Das ist in der Regel gut aufgegangen. Von manchen Beiträgen hätte ich mir etwas mehr allgemeinen Ertrag gewünscht, etwa zu der Grundidee europäischer Kommunikationsgeschichte, zu etablierten Kategorien oder Quellenproblemen. Der Beitrag von Andreas Fickers, Dana Mustata und Anne-Katrin Weber beispielsweise hinterfragt den Begriff der Fernsehnationen, löst sich vom nationalstaatlichen Denken und sucht nach übergreifenden Strukturprinzipien. Das mag nicht bei allen Themen angemessen sein und funktioniert hier auch deshalb, weil die einzelnen Ländergeschichten des Fernsehens schon gut erforscht sind.

Der Beiträge liefern einen differenzierten Blick auf den innereuropäischen Raum. Der Einbezug von Russland hilft, den Automatismus zu überwinden, bei Europa nur an die gegenwärtigen Grenzen der Europäischen Union zu denken. Bezüge in andere Teile der Welt findet die Leserin kaum, zumindest kaum über den US-amerikanischen Kontext hinaus. In diesem Zusammenhang ist es sicher auch zeitgetrieben, wenn ich beispielhaft auf die Verbindungen zwischen Kommunikationsgeschichte und europäischer Kolonialgeschichte hinweise. 
Aus Sicht einer „tiefen Internationalisierung“ kann man zwei Aspekte der Einleitung diskutieren: Zum einen bleibt die Begründung einer europäischen Kommunikationsgeschichte, bei allen Bekenntnissen zu Perspektivenpluralität, letztlich doch den etablierten Ideen von Aufklärungsphilosophen und Öffentlichkeitssoziologen aus Westeuropa und den USA verhaftet (etwa Weber, Park, Lasswell, Mills, Habermas, Durkheim, Tönnies). Zum anderen kann man das Projekt einer „Großerzählung“ diskutieren, das trotz (oder gerade aufgrund) der erkannten Vielfalt deutlich mitschwingt. Das Herausgeberteam argumentiert hier auch politisch und kreist immer wieder um die Europäische Union. Die Kommunikationsgeschichten, die in den Kapiteln aus Europa erzählt werden, wecken aber auch so Neugier. Ein gemeinsames Narrativ brauchen sie eigentlich nicht.

Das Rezept aus Teams und Länderauswahl ist aufgegangen, ebenso wie das Vorhaben, länderübergreifende und vergleichende Ansätze miteinander zu verbinden. Der Band bietet eine einzigartige kommunikationshistorische Zusammenschau und füllt eine bedeutsame Lücke. Denn das zusammengetragene Wissen hilft, Kommunikationsgeschichten in Beziehung zueinander zu setzen, Annahmen von Besonderheit und Differenz zu prüfen und behauptete Gemeinsamkeiten zu widerlegen. Es ist nun sehr einfach, sich über die Grenzen eines Landes hinweg mit der Medien- und Kommunikationsgeschichte in anderen Teilen Europas vertraut zu machen. Inhaltsverzeichnis und Indexe erlauben einen schnellen Zugriff auf Themen und Namen.

Funding Open Access funding enabled and organized by Projekt DEAL.

Open Access Dieser Artikel wird unter der Creative Commons Namensnennung 4.0 International Lizenz veröffentlicht, welche die Nutzung, Vervielfältigung, Bearbeitung, Verbreitung und Wiedergabe in jeglichem Medium und Format erlaubt, sofern Sie den/die ursprünglichen Autor(en) und die Quelle ordnungsgemäß nennen, einen Link zur Creative Commons Lizenz beifügen und angeben, ob Änderungen vorgenommen wurden.

Die in diesem Artikel enthaltenen Bilder und sonstiges Drittmaterial unterliegen ebenfalls der genannten Creative Commons Lizenz, sofern sich aus der Abbildungslegende nichts anderes ergibt. Sofern das betreffende Material nicht unter der genannten Creative Commons Lizenz steht und die betreffende Handlung nicht nach gesetzlichen Vorschriften erlaubt ist, ist für die oben aufgeführten Weiterverwendungen des Materials die Einwilligung des jeweiligen Rechteinhabers einzuholen.

Weitere Details zur Lizenz entnehmen Sie bitte der Lizenzinformation auf http://creativecommons.org/ licenses/by/4.0/deed.de.

Dr. Maria Löblich ist Professorin für Kommunikationsgeschichte und Medienkulturen an der Freien Universität Berlin. 